Communications in Physics, Vol. 23, No. 2 (2013), pp. 107-119

\title{
MAPPING ATOMIC HYDROGEN IN THE DISK OF THE MILKY WAY
}

\author{
NGUYEN VAN HIEP, PHAM TUAN ANH, PIERRE DARRIULAT, PHAM NGOC \\ DIEP, PHAM NGOC DONG, DO THI HOAI, PHAM THI TUYET NHUNG AND \\ NGUYEN THI THAO \\ VATLY, Institute for Nuclear Science and Technology, \\ 179 Hoang Quoc Viet, Cau Giay, Hanoi, Vietnam \\ Email: dieppn@vinatom.gov.vn
}

Received 19 December 2012; revised manuscript received 28 March 2013

Accepted for publication 08 May 2013

\begin{abstract}
The VATLY radio telescope has been used to draw the map of atomic hydrogen gas in the disk of the Milky Way. Effects resulting from its differential rotation, its cloud and arm structure and the presence of a dark matter halo have been observed.
\end{abstract}

\section{INTRODUCTION}

The Milky Way, which hosts the Sun, is a spiral galaxy that contains a black hole of nearly 4 million solar masses in its centre, a large central bulge, a relatively young and thin disk and a halo. The dark matter halo dominates the mass of the Galaxy and the luminous halo includes some 150 globular clusters believed to have formed at the same time as the Galaxy, over ten billion years ago, and very diffuse, hot, highly ionized gas extending out to some hundred kpc (1 pc=3.26 lyr). The central bulge hosts old Population II stars with a bar cutting across it.

The disk, a flat rotating system, contains atomic (HI) and molecular $\left(\mathrm{H}_{2}\right)$ hydrogen gas, dust, and stars. The Sun, about five billion years old, sits $\sim 2 / 3$ of the way from the centre to the edge of the disk $(\sim 8.3 \mathrm{kpc})$ and revolves around the centre of the Galaxy once every $\sim 250$ million years. The disk has been observed at all wavelengths. It is $\sim$ $300 \mathrm{pc}$ thick and $\sim 30 \mathrm{kpc}$ in diameter and is structured in spiral arms associated with density waves that trigger the birth of new stars. In addition to intermediate age stars such as the Sun, it contains brighter Population I objects, in the form of young, hot stars, stellar associations, open clusters, diffuse nebulae, and the bulk of the interstellar matter from which future stars will form.

Many recent observations have added unexpected features to the above overall picture of the Milky Way. In 2005, the Spitzer Space Telescope showed that the central bar extends farther out from the centre of the Galaxy than previously suggested and, in 2008, from a survey encompassing 110 million stars, made a strong case for the dominance 
of two major spiral arms joining neatly with the ends of the central bar: the ScutumCentaurus and Perseus arms, with the greatest densities of both young, bright stars, and older, red-giant stars [1]. Two minor arms, Sagittarius and Norma, are filled with gas and pockets of young stars.

Recent data from Fermi-LAT [2] have revealed two large gamma-ray bubbles, extending 50 degrees above and below the Galactic centre, with a width of about 40 degrees in longitude, spatially correlated with microwave and X-ray features observed by WMAP and ROSAT, respectively. They were possibly created by past accretion onto the central black hole or by a recent $(\sim 10 \mathrm{Myr})$ nuclear starburst.

Measurements of the Hydrogen alpha line [3] show nearby structures of gas that project far out of the plane of the Galaxy and that are lit up by ultraviolet photons from young stars.

In 2003, stars were discovered around the Milky Way [4] suggesting the presence of a complete outer torus encircling its disk ( $\sim 30 \mathrm{kpc}$ across). It would have a width of some $3 \mathrm{kpc}$, a thickness at the kpc scale and would contain between 100 million and 10 billion stars. It rotates at about $110 \pm 25 \mathrm{~km}$ per second (the Sun moves in the same direction at twice this speed). It might be the remains of a satellite galaxy, spun apart by the stronger gravity of the Milky Way.

Finally, in 2004 a new galactic arm of neutral hydrogen gas was discovered very close to the outer ring [5]. It may be a remnant from an earlier spiral arm.

Dynamic evidence for dark matter is ubiquitous in the Universe. A possible scenario [6] for the formation of the Milky Way implies the original gathering of a sufficient amount of dark matter that attracted ordinary matter, mostly hydrogen and helium gas. Stars formed and eventually piled up at the core, forming the galaxy. In 2007 the first threedimensional map of the large-scale distribution of dark matter in the universe was drawn using Huble Space Telescope data and weak gravitational lensing [7]. This new map provides the best evidence to date that normal matter, largely in the form of galaxies, accumulates along the densest concentrations of dark matter.

The Milky Way's dark halo is believed to outweigh galactic normal matter by a factor of $\sim 20$ and to extend $100 \mathrm{kpc}$ out from the galactic centre. Simulations [8] predict that there should have been over hundred separate "subhaloes" of dark matter within the overall galactic halo, each of the order of at least a kpc across, which should have attracted ordinary matter to form star clusters, $\sim 120$ of which should have become large enough to make dwarf galaxies. But only 15 dwarf satellite galaxies have been identified around the Milky Way thus far.

Dwarf satellite galaxies disturb the dark matter halo and cause the disk to warp. In 2007, a study of the warp caused by the two Magellanic Clouds suggested that the Milky Way's combined dark and visible mass must be twice as much as originally thought [9]. In 2010, the shape of the dark matter halo was deduced from the path of a dwarf galaxy whose stars have been "shredded" by the dark matter halo into a long tidal stream detected using red giant stars from the Two-Micron All Sky Survey [10].

The present article describes measurements of the HI content of the Milky Way disk performed using the VATLY radio telescope in Hanoi. 


\section{PRINCIPLE OF THE METHOD}

We used the VATLY radio telescope to measure the dependence on galactic longitude of the power flux density of the Milky Way disk integrated over the $21 \mathrm{~cm}$ hydrogen line.

The VATLY radio telescope has been described in previous publications [11] where its performance was shown to be excellent. The telescope is equipped with a mobile parabolic dish, $2.6 \mathrm{~m}$ in diameter, remotely adjustable in elevation and azimuth and is equipped for observation at frequencies in the region of the $21 \mathrm{~cm}$ hydrogen line. The hydrogen line signals the presence of hydrogen clouds in the field of view and is associated with electron spin flip in the hydrogen atom, while the continuum signals the presence of ionized matter and is mostly associated with synchrotron emission. Standard data collection consists in a sequence of successive measurements of $\sim 7.7 \mathrm{~s}$ duration each, digitized in the form of a frequency histogram covering $\sim 1.2 \mathrm{MHz}$ in 156 bins of $\sim 7.8$ $\mathrm{kHz}$ each, obtained by stitching together three independent $500 \mathrm{MHz}$ bandwidths. Super heterodyne uses a local oscillator frequency range of 1370 to $1800 \mathrm{MHz}$ and an intermediate frequency centred on $800 \mathrm{kHz}$ with a $6 \mathrm{~dB}$ range of 0.5 to $3 \mathrm{MHz}$. The back end includes analog to digital conversion on a dedicated PCI card, data being transferred to a hard disk for off-line analysis. The noise temperature was measured to be $\sim 140 \mathrm{~K}$.
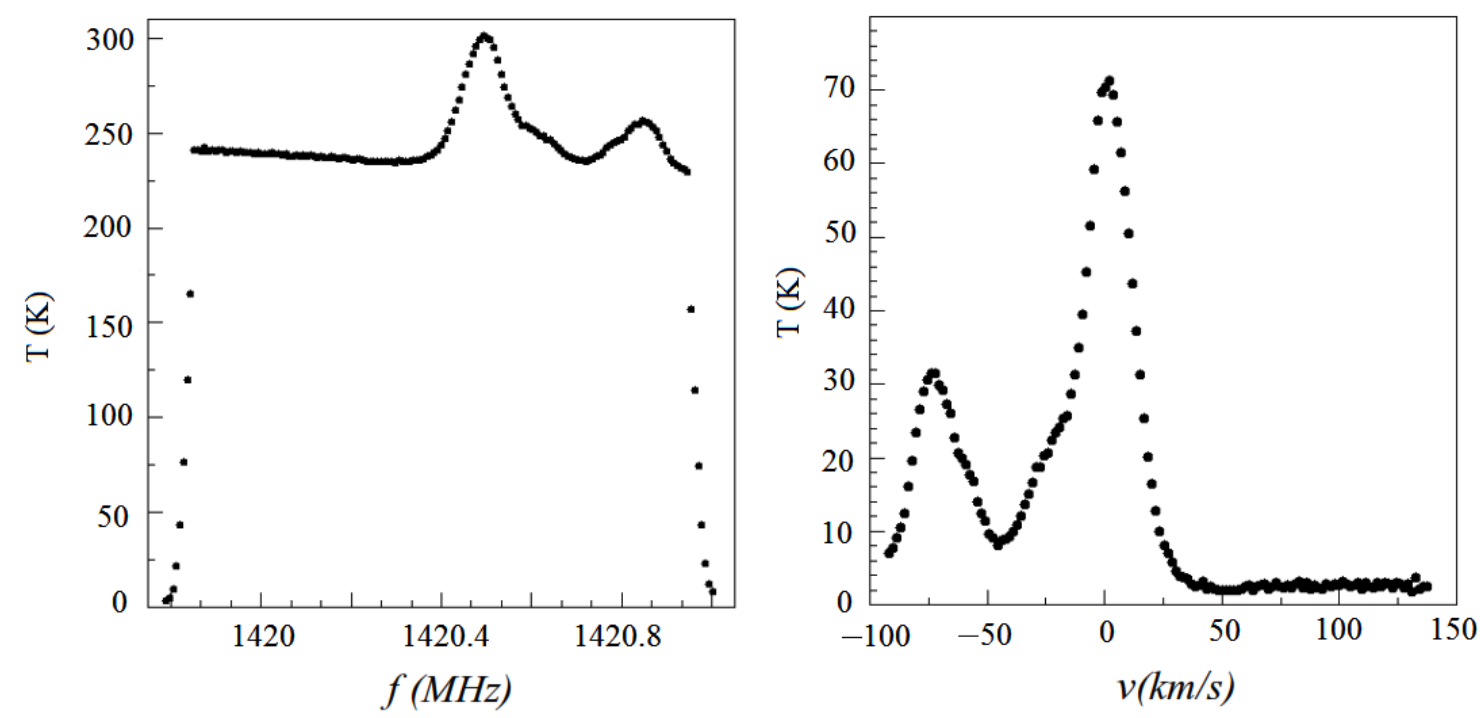

Fig. 1. A typical spectrum at $70^{\circ}$ galactic longitude. The received signal is shown as a function of frequency $(\mathrm{MHz})$ on the left panel and as a function of velocity with respect to the Sun $(\mathrm{km} / \mathrm{s})$, after continuum subtraction, on the right panel.

A pointing accuracy of $\sim 0.3^{\circ}$ is obtained after applying pointing corrections of the order of a degree. The size of the beam (FWHM) was obtained from drift scans of the Sun [11] to be $5.5 \pm 0.3^{\circ}$, corresponding to $\sigma=2.3 \pm 0.1^{\circ}$. 
The relative measurement uncertainty obtained for a single frequency bin by summing $n$ successive measurements has the form $\Delta P / P=\sqrt{(0.27 \%)^{2}+(1.59 \%)^{2} / n}$, giving an optimal time scale of $\sim 10 \mathrm{mn}$ per measurement.

A set of 117 spectra have been recorded between February $22^{\text {nd }}, 2012$ and March $9^{t h}, 2012$ of the visible part of the disk of the Milky Way. Each spectrum was taken by pointing to a point of zero galactic latitude and fixed galactic longitude during $15 \mathrm{mn}$. The galactic longitude was chosen in steps of $2.5^{\circ}$ (of the order of one half of the beam width) from $20^{\circ}$ to $270^{\circ}$. Pointing corrections were systematically applied. A typical spectrum is shown in Fig. 1 (left).

The spectra display a peak structure as expected from the combined effect of the presence of distinct clouds and of velocity crowding producing a peak at the terminal velocity: the cloud velocities around the galactic centre, together with the cloud peculiar motions, cause a Doppler shift of the $21 \mathrm{~cm}$ line that differs from cloud to cloud. Each peak is associated with an atomic hydrogen cloud and the measurement of its frequency provides a measurement of its velocity with respect to the Sun.

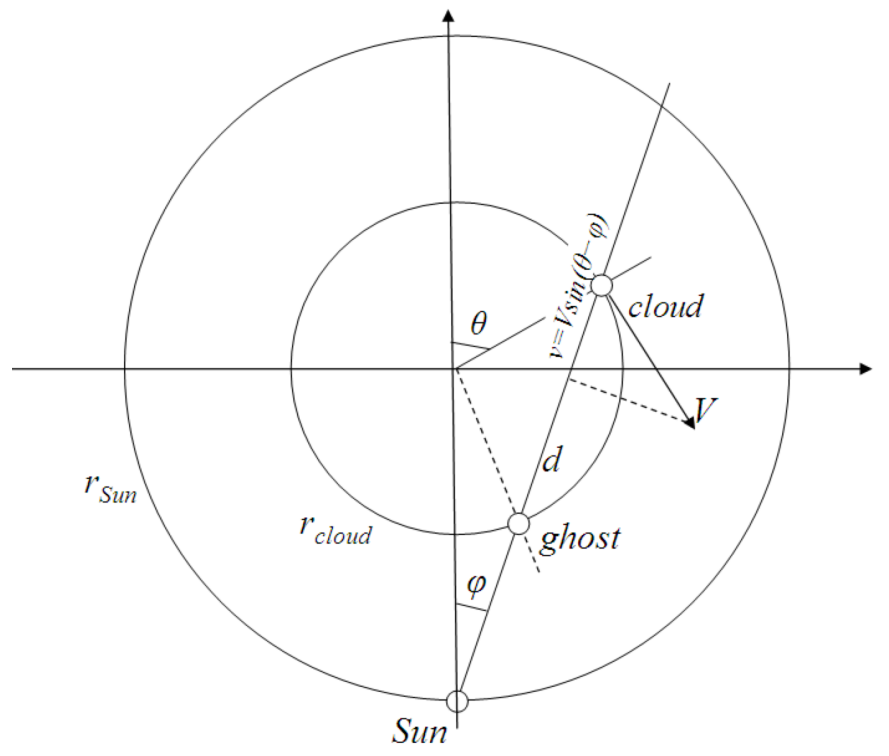

Fig. 2. Geometry of the cloud mapping problem in a configuration such that $r_{\text {cloud }}<r_{\text {Sun }}$.

Knowing the Galaxy rotation curve, it should be straightforward, once the velocity of such a cloud is known, to calculate its position along the line of sight, at a distance $d$ from the Sun. Indeed, as can be seen from Fig. 2,

$$
v=V\left(r_{\text {cloud }}\right) \sin (\theta-\varphi) \text { and } r_{\text {cloud }} / \sin \varphi=r_{\text {Sun }} / \sin (\theta-\varphi)=d / \sin \theta
$$

Here, $r_{S u n}$, the distance of the Sun to the centre of the Galaxy, is known; $v$ and $\varphi$ are measured; the function $V$ (the rotation curve relative to the Sun rotation), is supposed to be known. One is left with three unknowns $\left(d, \theta\right.$ and $\left.r_{\text {cloud }}\right)$ and three equations. However, as is obvious from Fig. 2, changing $\theta$ into $\pi-\theta+2 \varphi$ and $d$ into $d-2 r_{\text {cloud }} \cos (\theta-\varphi)$ gives 
another solution, marked "ghost" in the figure. In the case illustrated, where $r_{\text {cloud }}<r_{\text {Sun }}$, there are indeed two possible solutions and the position of the cloud cannot be measured unambiguously. On the contrary, when $r_{\text {cloud }}>r_{\text {Sun }}$, there is only one solution, the ghost being now at opposite direction from the line of sight.

\section{THE GALAXY ROTATION CURVE}

The rotation curve of a galaxy displays the dependence of the orbital circular velocity of stars or gas clouds on their distance from the centre. The manner in which the velocities vary with radius reflects the distribution of mass in the galaxy: for a solid disk, the velocity is proportional to radius; in a galaxy in which most of the mass is concentrated at the centre, the velocity decreases with the square root of the radius, this behaviour is known as "Keplerian decline"; a flat rotation curve, that is one in which the velocity is constant over some range of radii, implies that the mass is increasing linearly with radius (within a homogeneous medium it increases as the cube of the radius and in a disk, as the square). Most galaxies have rotation curves that show solid body rotation in the very centre, followed by a slowly rising or constant velocity rotation in the outer parts. Very few galaxies show any evidence for Keplerian decline.

The first rotation curves derived for the whole Milky Way used HI [12] and CO [13] data. The rotation of the outer Galaxy was later studied by direct measurement of the distances to the stars in optical HII regions [14] and by CO observations of the molecular clouds in the HII regions [15]. Here, we use a rotation curve established for the northern Milky Way [16] on the basis of the above data and of additional CO data [17]. It also uses an evaluation of the mass of the Galaxy, up to $60 \mathrm{kpc}$ radius, deduced from globular cluster data [18]. It is displayed in Fig. 3 (left).
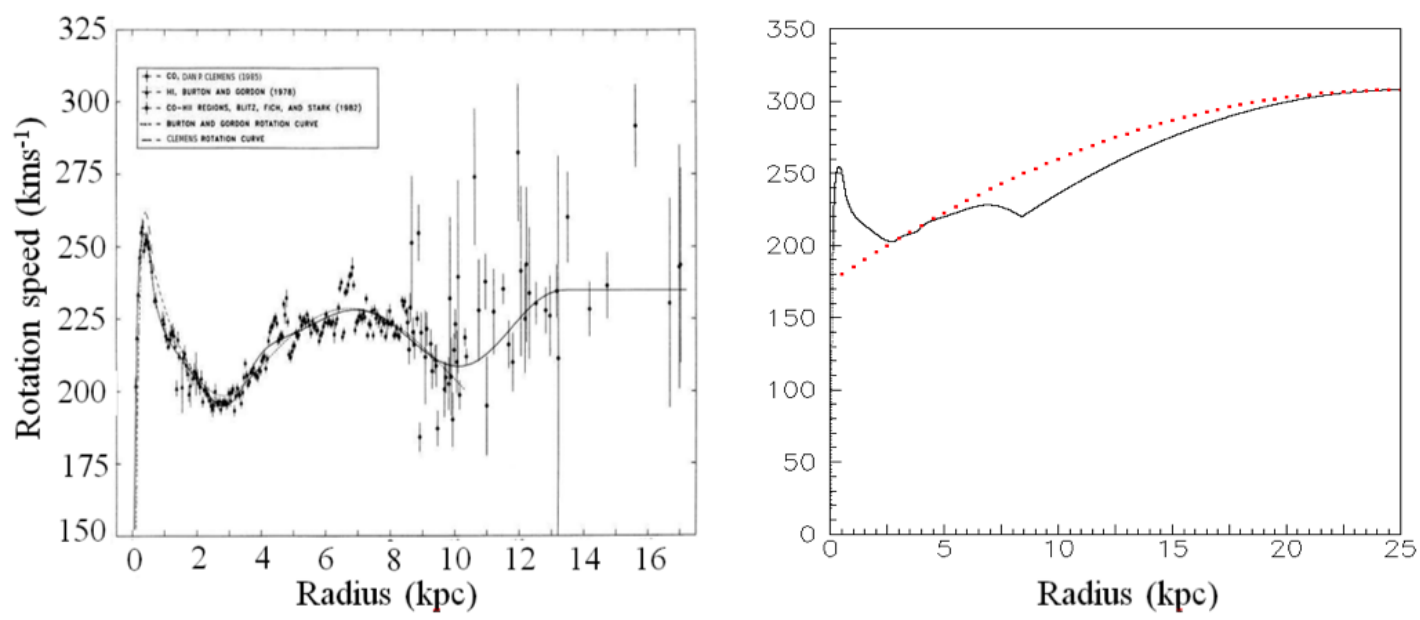

Fig. 3. Rotation curve from Reference 16. Data are $\mathrm{HI}$ for radii smaller than $\mathrm{r}_{\text {Sun }}$ and CO for radii larger than $\mathrm{r}_{\text {Sun }}$. The right panel displays the rotation curve used in the present work. 
From the Sun outward, we take a quadratic dependence on radius of the form $v(r)=v_{\text {Sun }}\left[1+0.1\left(5-r / r_{\text {Sun }}\right)\left(r / r_{\text {Sun }}-1\right)\right]$ where $r_{\text {Sun }}=8.4 \mathrm{kpc}$ and $v_{\text {Sun }}=220 \mathrm{~km} / \mathrm{s}$. It reaches its maximum at $3 r_{\text {Sun }}$, beyond which it is assumed to remain constant. As can be seen on Fig. 3 (left), the CO data leave much freedom at radii larger than $r_{S u n}$. However, we observed that at galactic longitudes of the order of $50^{\circ}$ and $110^{\circ}$ velocities reaching $300 \mathrm{~km} / \mathrm{s}$ were necessary to reproduce the spectra. This motivated the choice displayed in Fig. 3 right.

\section{EVIDENCE FOR DIFFERENTIAL ROTATION}

On each measured frequency spectrum, a constant continuum has been subtracted and the extreme frequency bins have been set to zero in order to avoid end effects. Conversion of the frequency scale $(f)$ into a velocity scale $(v)$ was made using the Doppler formula: $v=c\left(f_{0}-f\right) / f_{0}-v_{\text {Sun }}$, where $c$ is the light velocity and $f_{0}$ the rest frequency of $1.42 \mathrm{GHz}$. Here the velocity is projected along the line of sight, red shifts (away from the Earth) corresponding to positive velocities and blue shifts (toward the Earth) to negative velocities. The quantity $v_{\text {Sun }}$ that has been subtracted accounts for the peculiar Sun velocity, projected on the line of sight, $39 \cos \left(197^{\circ}-l\right) \mathrm{km} / \mathrm{s}, l$ being the galactic longitude. In addition to its peculiar motion, the Sun rotates around the centre of the Galaxy with a velocity of $220 \mathrm{~km} / \mathrm{s}$. In what follows, we shall work in the rotating frame where any object rotating at the same angular velocity as the Sun is at rest. Namely, at a distance $r$ where the rotation curve predicts a velocity $v(r)$, we shall use a relative velocity $v^{\prime}(r)=v(r)\left(1-r / r_{\text {Sun }}\right)$.

Fig. 1 (right) shows the result of these operations in the case of the raw spectrum displayed in Fig. 1 (left). The obtained spectra may contain one or several peaks, resolved or not: Fig. 4 displays three typical configurations.
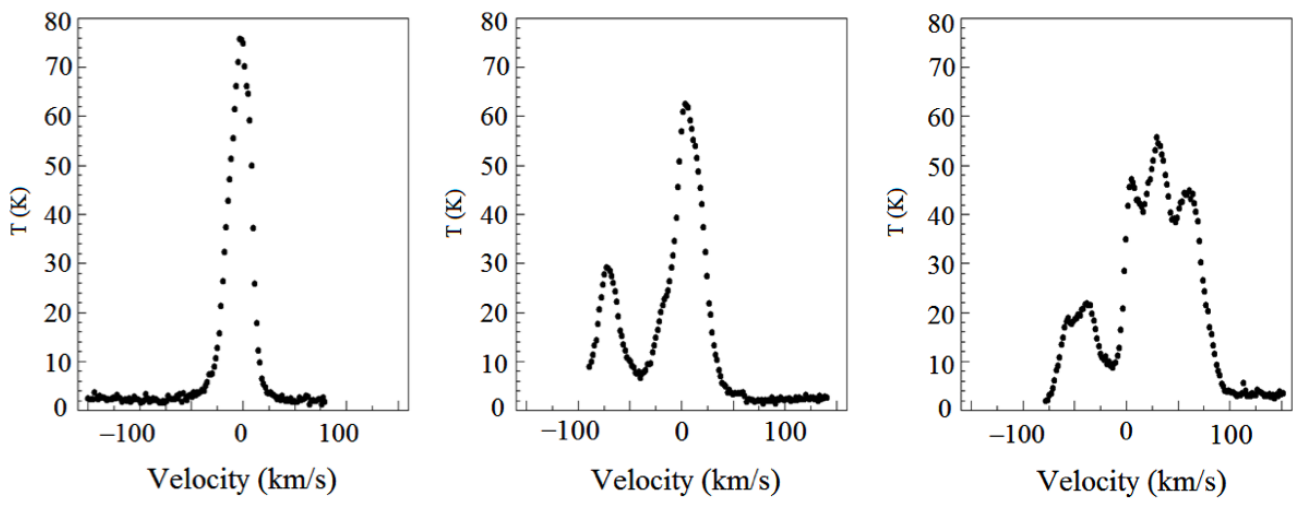

Fig. 4. Three typical spectra at $180^{\circ}, 65^{\circ}$ and $40^{\circ}$ galactic longitude respectively showing one, two or several peaks.

Fig. 5 displays our measurements in the $(\varphi, v)$ plane where $v$ is the measured radial velocity and $\varphi=2 \pi-l$. It is compared with other measurements [19]. Also shown is the prediction for a uniform disk of radius $25 \mathrm{kpc}$ obeying the rotation curve displayed 
in Fig. 3. Replacing the low radius part of the rotation curve by a simple proportion, $v(r)=v_{\text {Sun }} r / r_{\text {Sun }}$, would suppress positive velocities at small galactic latitudes. The observed sine wave is very strong evidence for a global differential rotation (a solid disk would mean zero velocities at all galactic longitudes).
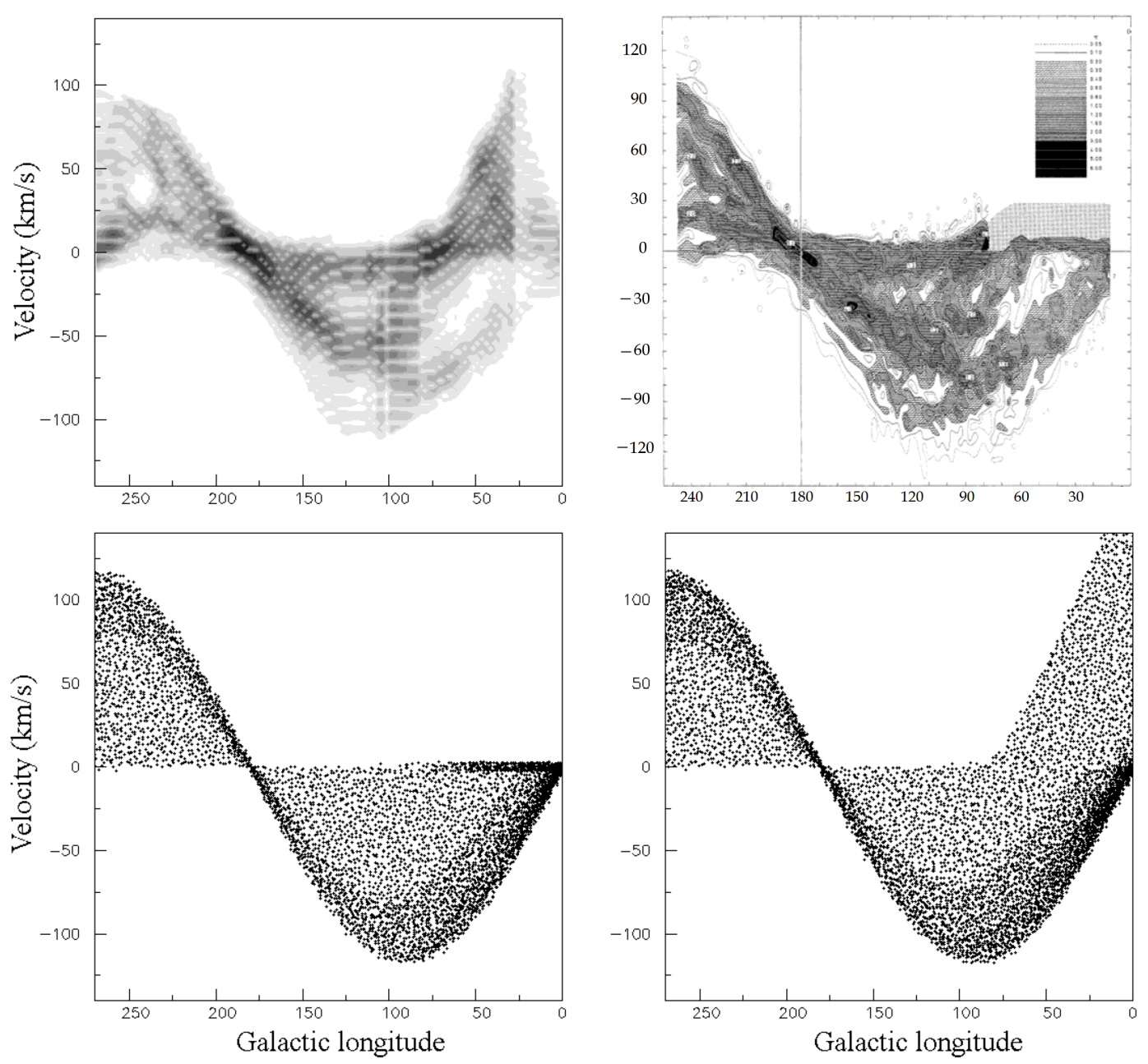

Fig. 5. Distributions in the $(\varphi, v)$ plane. Upper left panel: present measurements; upper right panel: measurements from Reference 19; lower right panel: prediction for the rotation curve shown in Figure 3 and used in the present work; lower left panel: prediction for a modified rotation curve obtained from the preceding one by using a form $v(r)=v_{\text {Sun }} r / r_{\text {Sun }}$ for radii smaller than the Sun radius. 


\section{EFFECT OF THE CLOUD AND ARM STRUCTURE}

In a first phase, each spectrum was individually inspected and resolved into a series of peaks with emphasis on the peak velocities rather than on their amplitudes. If one could ignore the peculiar motions of the HI clouds, all spectra would be made of lines. The only broadening factors would be the motion of the hydrogen atoms within the clouds and the instrumental resolution, which contributes $\sim 0.8 \mathrm{~km} \mathrm{~s}^{-1}$. Including the peculiar motions of the clouds and their possible rotation on themselves broadens and/or shifts such lines. We make the simplifying assumption that, on average, the directions of the peculiar velocities can be considered as being random and their Cartesian coordinates as Gaussian distributed around the origin, resulting, on average, in a broadening of the observed lines. In most cases, resolving the velocity spectra into a series of peaks is a simple exercise, but in a few cases it is unclear whether one is dealing with a single peak or with two nearly superimposed peaks, leaving some subjectivity in the definition of the peak list. Four typical examples are illustrated in Fig. 6.

For each peak, a $d$ interval was evaluated on the line of sight, within which the HI source needs to be confined in order to contribute to the peak. In ambiguous cases, two such intervals were evaluated. This was made by finding the best fit to the resulting velocity distribution, smeared using a Gaussian having a $\sigma$ of $14 \mathrm{~km} / \mathrm{s}$, chosen adhoc to well describe, on average, the observed line widths. As two clouds of same absolute HI luminosity located at different distances on the line of sight have different apparent luminosities, the far away cloud being fainter by a factor equal to the square of the ratio of their respective distances, a weighting factor $\left(d / r_{S u n}\right)^{2}$ has been applied to draw the map displayed in Fig. 7.

A clear arm structure, resulting from the continuity of the peak configuration between neighbour sectors of galactic longitude, is visible. It is strongly correlated with the known arm structure [20], with important concentrations of clouds along the ScutumCentaurus and Perseus arms. However, the real map is expected to be very much smeared with respect to what is obtained here because the $d$ intervals are much narrower than the $d$ region covered by the peak.

For this reason, in a second phase, having now compared the measured map with the known arm structure, we attempt to draw a more realistic HI map of the Milky Way disk. We do not need any longer to identify peaks: the non ambiguous cases provide a direct mapping of the measured signal onto the galactic disk, each velocity bin being associated with a unique distance bin in the galactic longitude interval being considered. However, for this to be true, we need to use a monotonous rotation curve in order to ensure that a given velocity be associated with one and only one distance to the Galaxy centre. The form used for this purpose, illustrated in Fig. 3 (right), is $v(r)[\mathrm{km} / \mathrm{s}]=308-0.213(r[\mathrm{kpc}]-25)^{2}$. Drawing the map implies transforming the velocity distribution into a $d^{2}$ distribution using the proper Jacobian and using weights $\left(d / r_{\text {Sun }}\right)^{2}$ to transform from apparent to absolute brightness. Fig. 8 displays the result. Despite considerable smearing, the Perseus and Scutum-Centaurus arms are still visible. For galactic longitudes in the $\left[0^{\circ}, 90^{\circ}\right]$ quadrant, the evaluation of $d$ is ambiguous: we show separate maps, one using the smaller $d$ value and the other the larger. The qualitative nature of the exercise must be kept in mind: 

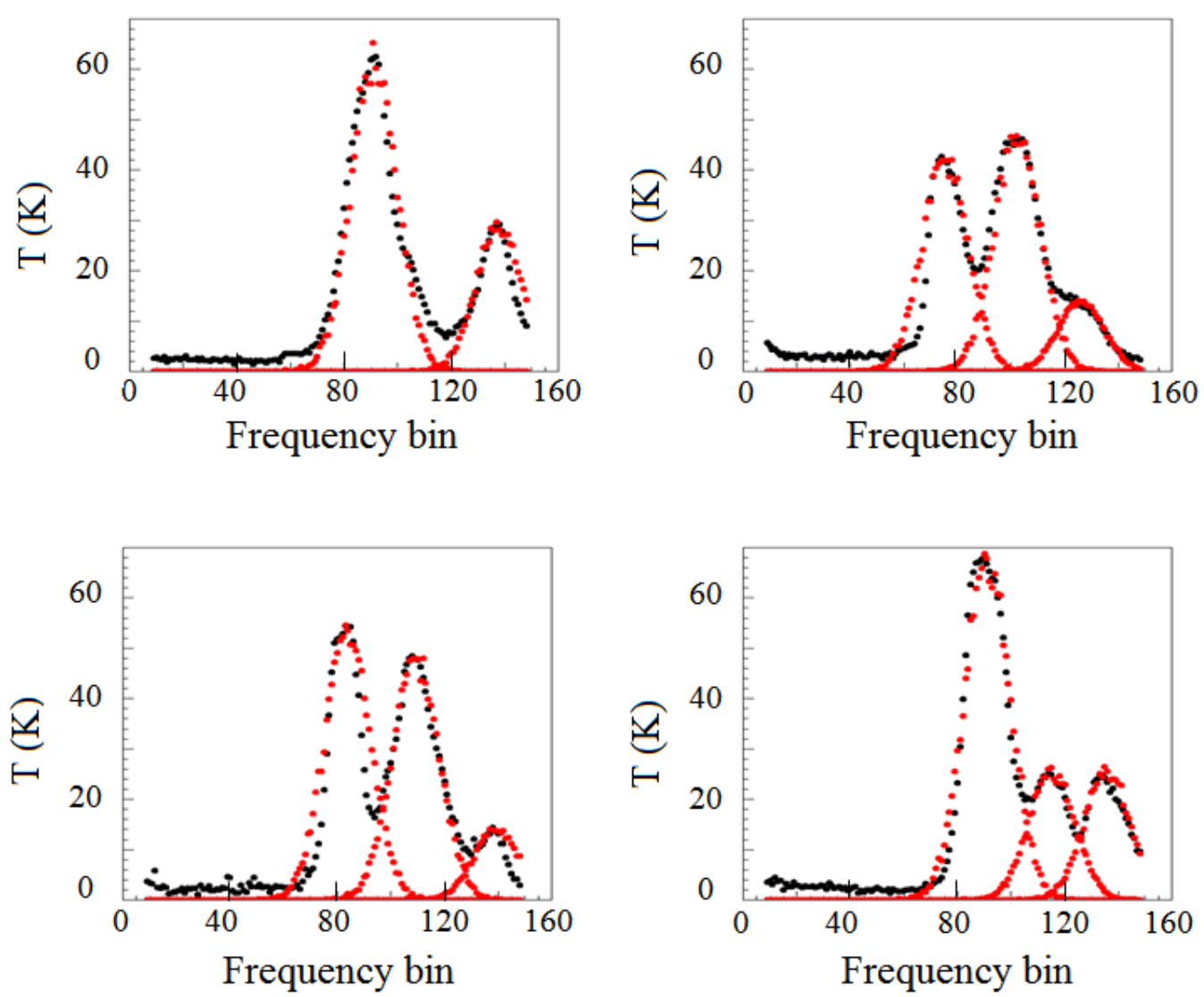

Fig. 6. Four examples of frequency distributions showing the definition of peaks. Measurements (black) are compared with the peak associated with the adopted cloud locations according to the algorithm described in the text. At this phase of the analysis, emphasis is on evaluating peak positions rather than amplitudes.

the crudeness of the rotation curve being used and the fact that at large distances a small error in $v$ causes a large error in $d$ prevent a serious quantitative evaluation to be obtained.

\section{EFFECT OF THE PRESENCE OF DARK MATTER}

The coherence of the results obtained in the preceding sections using a rotation curve that stays high at large distances is an indirect effect of the presence of dark matter at large distances. We now illustrate the effect of dark matter more directly, without making use of any a priori rotation curve.

In the absence of dark matter, and in the approximation of the whole galactic mass being concentrated at the galactic centre, the rotation curve would take the form $v=v_{\text {Sun }} \sqrt{r_{\text {Sun }} / r}$ with $r_{\text {Sun }}=8.4 \mathrm{kpc}$ and $v_{\text {Sun }}=220 \mathrm{~km} / \mathrm{s}$. In particular, at galactic 

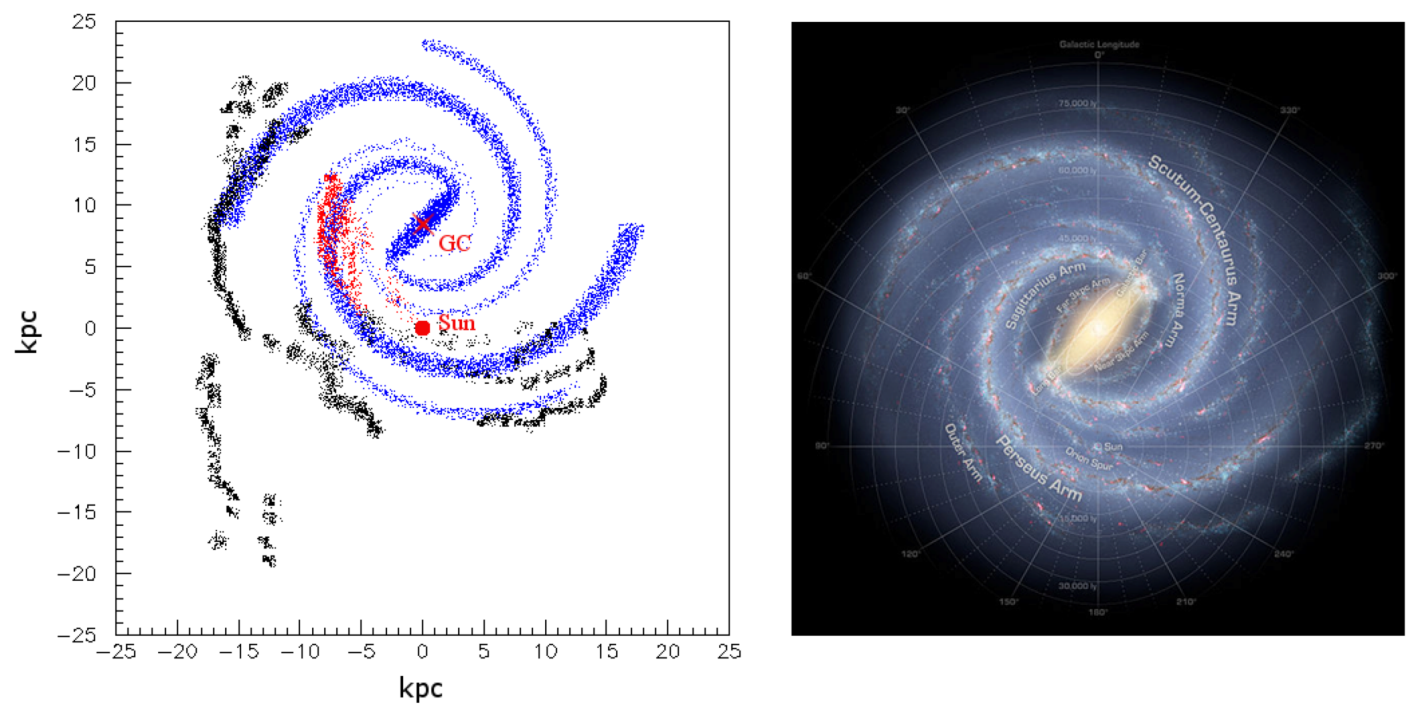

Fig. 7. Left panel: map obtained by tracking the velocity peaks (see text). Red dots mark ambiguous sources. The known arm structure as found by Spitzer [1] is shown in blue and independently displayed in the right panel.
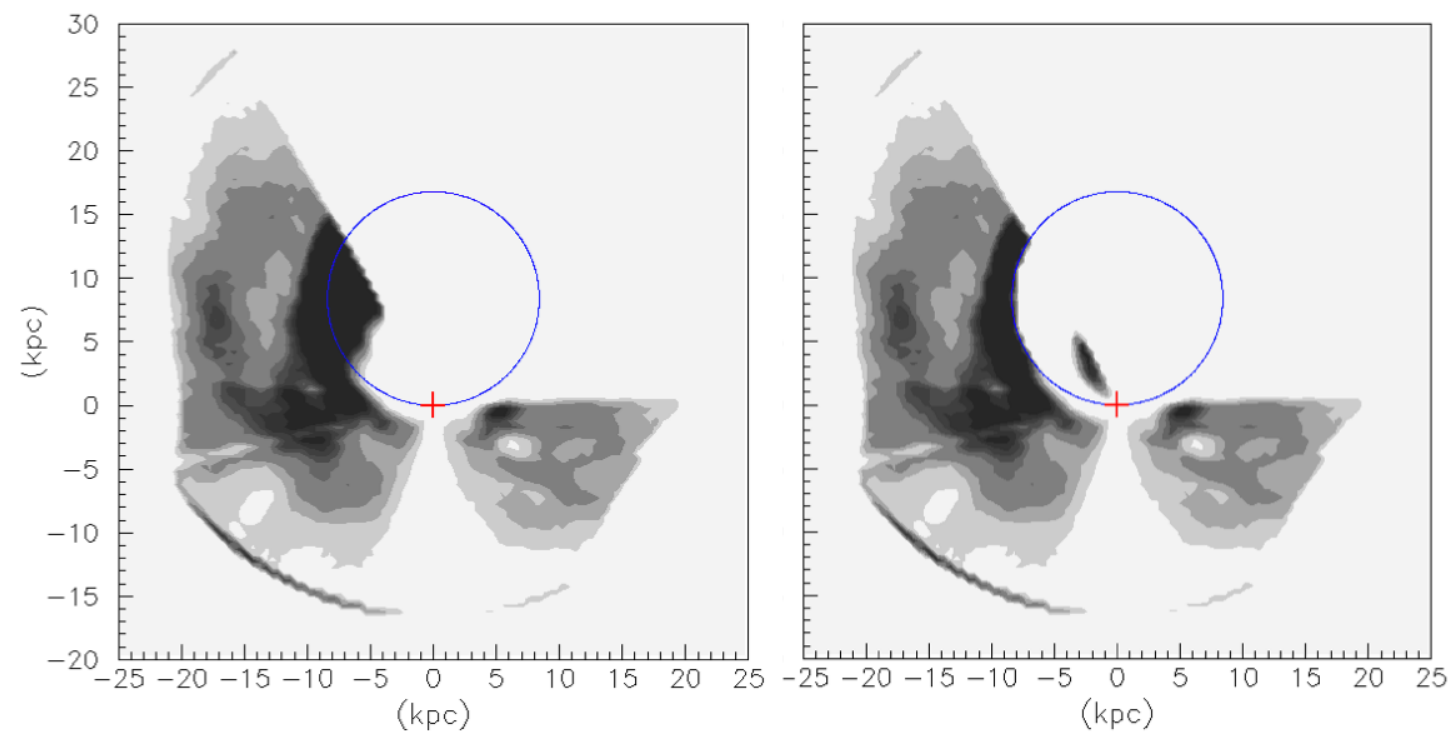

Fig. 8. Measured HI map of the disk using weights $\left(d / r_{\text {Sun }}\right)^{2}$. The circle centred on the centre of the Galaxy and passing by the Sun is shown in black. Its inside is associated with ambiguous cases, its outside with non-ambiguous cases. Ambiguous cases are displayed separately in the two panels, farther away from the Sun on the left and closer up on the right. 
longitude $l$ such that $|l|<90^{\circ}, r$ must exceed $r_{\text {min }}=r_{\text {Sun }}$ sinl, corresponding to the line of sight being tangent to the circle of radius $r_{\text {min }}$.

In such a tangent configuration, the rotation and recession velocities are equal. Therefore, in the absence of dark matter, the recession velocity would not exceed

$$
v_{\text {Sun }} \sqrt{r_{\text {Sun }} / r_{\text {min }}}-v_{\text {Sun }} r_{\text {min }} / r_{\text {Sun }}=v_{\text {Sun }}(1 / \sqrt{\operatorname{sinl}}-\operatorname{sinl})
$$

where the second term subtracts the rotation of the Sun. Fig. 9 (left) displays the dependence of the measured terminal velocities on $r_{\min }$ and compares them with $v_{\text {Sun }}(1 / \sqrt{\rho}-\rho)$ where $\rho=r_{\text {min }} / r_{\text {Sun }}$ to which they should tend when $r_{\text {min }}$ increases if there were no dark matter. In fact, they exceed this limit, giving a direct illustration of the presence of a dark matter halo at large distances from the galactic centre. The jump observed at $r_{\min } \sim 2.5$ $\mathrm{kpc}$ is associated with the appearance of a new arm of clouds, as illustrated in Fig. 9 (right). The Keplerian differential rotation curve reaches 0 at $r_{\min }=r_{\text {Sun }}$. Indeed, in this case, corresponding to $90^{\circ}$ galactic longitudes, all cloud velocities on the line of sight should be smaller than that of the Sun.
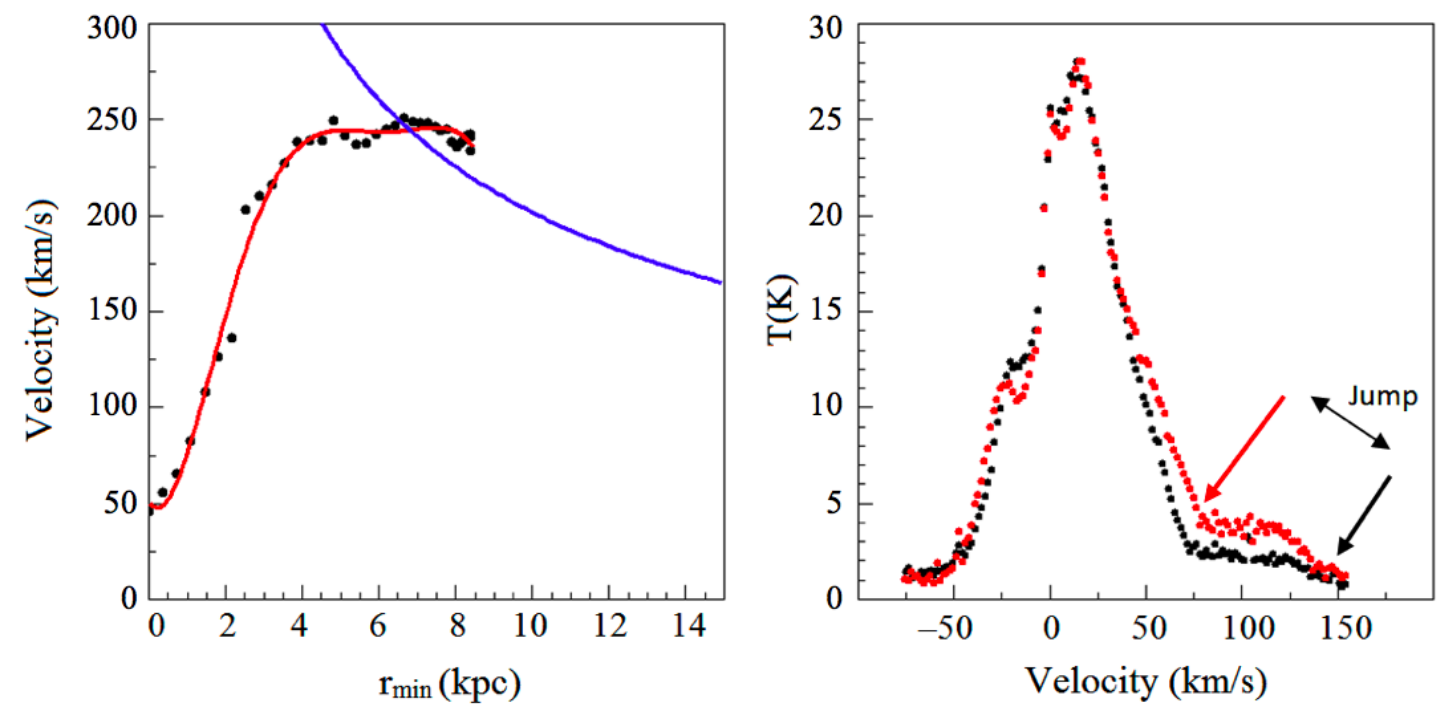

Fig. 9. Left: The measured terminal velocities (in $\mathrm{km} \mathrm{s}^{-1}$, Sun subtracted) are plotted as a function of $r_{\min }(\mathrm{kpc})$. The red line is a polynomial fit to the points. The blue line is the Keplerian differential rotation curve toward which the measured points should tend in the absence of dark matter. Right: The two differential velocity spectra on either side of the jump at $r_{\text {min }} \sim 2.5 \mathrm{kpc}$, showing the appearance of a new arm of $\mathrm{HI}$ clouds (arrows indicate the location of the respective end points).

\section{SUMMARY}

A map of atomic hydrogen (HI) in the disk of the Milky Way has been drawn in steps of $2.5^{\circ}$ of galactic longitude using the VATLY radio telescope tuned on the $21 \mathrm{~cm}$ 
hydrogen line. Clear evidence has been obtained for the differential rotation of the Galaxy and the effect of the clumping of atomic hydrogen in giant clouds has been observed. Good agreement has been achieved with the known spiral structure of the disk and with the rotation curve of Reference 16, which implies the existence of a dark matter halo. Indeed, the effect of the presence of dark matter has been directly illustrated by the dependence on galactic longitude of the measured terminal velocities, implying that the rotation curve significantly exceeds the Keplerian limit at large distances from the galactic centre.

The quantity and quality of the data that we have been able to collect in this early phase of operation has demonstrated the excellent performance of the telescope and its perfect suitability as a training tool. In a tropical country such as Vietnam, where the sky is very often obscured by fog and clouds, the teaching potential of such an instrument is far superior to that of an optical telescope. Future observations of other major radio sources, such as the Sun, nearby galaxies and SNRs, are now at hand.

\section{ACKNOWLEDGEMENTS}

We express our deep gratitude to the referee for comments that greatly helped in improving the quality of the manuscript. Financial and/or material support from the Institute for Nuclear Science and Technology, NAFOSTED, the World Laboratory, Rencontres du Vietnam and Odon Vallet fellowships is gratefully acknowledged.

\section{REFERENCES}

[1] R.A. Benjamin et al. (Spitzer), 630 (2005) L149; E.Churchwell et al., PASP 121 (2009) 213.

[2] M. Su, T.R. Slatyer and D.P. Finkbeiner, ApJ 724 (2010) 1044.

[3] E. Gonzalez-Solares et al., MNRAS 5388 (2008) 89.

[4] B. Yanny et al., (2003) ApJ 588 (2003) 824 and 605 (2004) 575; R.A. Ibata et al., Mon. Not. Roy. Astron. Soc. 340 (2003) L21.

[5] N.M. McClure-Griffiths et al., ApJ 607 (2004) L127.

[6] J. Diemand, M. Kuhlen and P. Madau, ApJ 657 (2007) 262.

[7] www.spacetelescope.org/images/heic0701b/.

[8] J.E. Taylor, J. Silk and A. Babul, Dark Matter in Galaxies, in ASP Conf. Series (2004), S. Ryder, D.J. Pisano, M. Walker and K. Freeman Editors.

[9] G. Besla et al., ApJ 668 (2007) 949 and ApJL 721 (2010) L97; X. Zhiang et al., arXiv:1209. 3856v1 [astro-ph. GA] 18 Sep 2012 and references therein.

[10] www.astronomy.org.nz, Auckland Astron. Soc. J. (2010) 16.

[11] N.V. Hiep et al., Comm. Phys. 22 (2012) 365.

See also: N.V. Hiep, Presented at the Second Academic Conference on Natural Science for Master and PhD students from Cambodia, Laos, Malaysia and Vietnam, 11-15 October 2011, Vinh, Vietnam.

P.T.Anh (on behalf of VATLY) presented at the 7th Annual Conference of the Thai Physics Society, May 9-12, 2012, Phranakhon Si, Ayutthaya, Thailand.

N.V.Hiep (on behalf of VATLY) presented at the 1st Southeast Asian Young Astronomers Collaboration (SEAYAC) Meeting, November 5-9, 2012, Puerto Princesa City, Palawan, Philippines.

[12] K.K. Kwee, C.A. Muller and G. Westerhout, Bull. Astr. Inst. Netherl. 12 (1954) 211.

[13] W.B. Burton and M.A. Gordon, A\&A 63 (1978) 7.

[14] A.F.J. Moffat, M.P. Fitzgerald and P.D. Jackson, Astronom. Astrophys. Suppl. 38 (1979) 19.

[15] L. Blitz, Ap. J. Lett. 231 (1979) 115; L. Blitz, M. Fich and A.A. Stark, Ap. J. Supp. 49 (1982) 183. 
[16] D.P. Clemens, The Astrophys. J. 295 (1985) 422.

[17] D.B. Sanders, D.P. Clemens, N.Z. Scoville and P.M. Solomon, Ap. J. Suppl. 60 (1986) 1.

[18] F.D.A. Hartwick and W.L.W. Sargent, Ap. J. 221 (1978) 512.

[19] P. O. Lindblad, The velocity distribution of atomic hydrogen in the outer parts of the galactic system according to the Kootwijk Survey 1953 - 55, Bulletin of the Astronomical Institutes of the Netherlands Supplement, Vol. 1, p.77. (1966).

[20] R.A. Benjamin et al., The Astrophys. J. 630 (2005) L149. 